\title{
Response to: Comment on "Does the Equivalence between Gravitational Mass and Energy Survive for a Composite Quantum Body?"
}

\author{
A. G. Lebed ${ }^{1,2}$ \\ ${ }^{1}$ Department of Physics, University of Arizona, 1118 E. 4th Street, Tucson, AZ 85721, USA \\ ${ }^{2}$ L. D. Landau Institute for Theoretical Physics, 2 Kosygina Street, Moscow 117334, Russia \\ Correspondence should be addressed to A. G. Lebed; lebed@physics.arizona.edu
}

Received 28 February 2017; Accepted 31 May 2017; Published 10 October 2017

Academic Editor: Shi-Hai Dong

Copyright (C) 2017 A. G. Lebed. This is an open access article distributed under the Creative Commons Attribution License, which permits unrestricted use, distribution, and reproduction in any medium, provided the original work is properly cited. The publication of this article was funded by SCOAP ${ }^{3}$.

\begin{abstract}
We have recently shown that both passive and active gravitational masses of a composite body are not equivalent to its energy due to some quantum effects. We have also suggested idealized and more realistic experiments to detect the above-mentioned inequivalence for a passive gravitational mass. The suggested idealized effect is as follows. A spacecraft moves protons of a macroscopic ensemble of hydrogen atoms with constant velocity in the Earth's gravitational field. Due to nonhomogeneous squeezing of space by the field, electron ground state wave function experiences time-dependent perturbation in each hydrogen atom. This perturbation results in the appearance of a finite probability for an electron to be excited at higher energy levels and to emit a photon. The experimental task is to detect such photons from the ensemble of the atoms. More realistic variants of such experiment can be realized in solid crystals and nuclei, as first mentioned by us. In his recent comment on our paper, Crowell has argued that the effect, suggested by us, contradicts the existing experiments and, in particular, astronomic data. We show here that this conclusion is incorrect and based on the so-called "free fall" experiments, where our effect does not have to be observed.
\end{abstract}

\section{Introduction}

I would like to thank Benjamin Crowell for his comment [1] on my article [2]. Creation of the so-called Theory of Everything is well known to be one of the most important problems in physics. It is also known that development of the Quantum Gravitation theory is one of the most important steps in this direction. Nevertheless, the latter problem appears to be extremely difficult. One of the reasons for that is the fact that the foundations of General Relativity and Quantum Mechanics are very different. Another reason is the absence of the corresponding experimental data. We recall that, so far, quantum effects have been directly tested only in the Newtonian variant of gravitation (see, e.g., $[3,4]$ ). In this complex situation, we have recently suggested two novel phenomena [2, 5-10]. In particular, we have demonstrated that both passive and active gravitational masses of a composite body are not equivalent to its energy due to some quantum effects. We have also suggested two experimental ways [2, 510] to test the above-mentioned phenomena. If one of such experiments is done, it will be the first direct observation of quantum effects in General Relativity.

\section{Goal}

In his recent comment [1], Crowell criticizes one of the experiments, suggested by us [2], which can demonstrate inequivalence between passive gravitational mass of a composite quantum body and its energy. The idealized variant of the experiment is as follows. There is a macroscopic ensemble of hydrogen atoms with each of them being in ground state at $t=0$. Protons of all atoms are dragged by a spacecraft with constant velocity in the Earth's gravitational field. Due to nonhomogeneous squeezing of space by the gravitational field, the atoms are shown $[2,5-8]$ to become excited and emit photons. As mentioned in [5], the above described 
phenomenon is very general and have to be observed in solids, nuclei, and elementary particles. The main criticism of the experiment $[2,5-8]$ in the comment [1] is the statement that the application of our theory to experiments on proton decay is not consistent with the existing experimental data. The goal of our reply is threefold. First, we pay attention that the discussed existing experimental data in [1] are obtained for free falling objects. On the other hand, the idealized experiment, suggested by us $[2,5-8]$, corresponds to transportation of centers of masses of the hydrogen atoms (i.e., protons) by spacecraft with a constant velocity. We stress that these are two different types of experiments. Second, to strengthen our arguments, we derive the Hamiltonian for the transportation of a hydrogen atom with constant velocity, semiquantitatively introduced in Refs. [2, 5-8], from the Dirac equation in a curved space-time of General Relativity. Third, we discuss "free fall" experiments for a hydrogen atom and make the conclusion that the effect, suggested by us for passive gravitational mass $[2,5-8]$, does not have to be observed under such conditions. Thus, proton decay does not have to demonstrate our effect in "free fall" experiments too. So, we make a conclusion that, contrary to the statement of the comment [1], the existing experiments on proton decay do not contradict our theoretical results.

\section{Semiquantitative Hamiltonian}

First, let us derive the Hamiltonian of [2, 5-8] for a hydrogen atom in the Earth's gravitational field, using semiquantitative approach. Below, we consider the case of a weak gravitational field; therefore, we can write the standard interval, describing space-time in a weak field approximation [11]:

$$
\begin{aligned}
d s^{2}= & -\left(1+2 \frac{\phi}{c^{2}}\right)(c d t)^{2} \\
& +\left(1-2 \frac{\phi}{c^{2}}\right)\left(d x^{2}+d y^{2}+d z^{2}\right), \\
\phi= & -\frac{G M}{R} .
\end{aligned}
$$

[Here $G$ is the gravitational constant, $c$ is the velocity of light, $M$ is the Earth mass, and $R$ is the distance between its center and proton.] In accordance with General Relativity, we introduce the so-called local proper space-time coordinates,

$$
\begin{aligned}
& x^{\prime}=\left(1-\frac{\phi}{c^{2}}\right) x, \\
& y^{\prime}=\left(1-\frac{\phi}{c^{2}}\right) y, \\
& z^{\prime}=\left(1-\frac{\phi}{c^{2}}\right) z, \\
& t^{\prime}=\left(1+\frac{\phi}{c^{2}}\right) t,
\end{aligned}
$$

where space coordinates do not depend on time and where the interval (1) has the Minkowski form.
In these local space-time coordinates, we can approximately write the Schrödinger equation for electron in the atom in the standard form,

$$
\begin{gathered}
i \hbar \frac{\partial \Psi\left(\mathbf{r}^{\prime}, t^{\prime}\right)}{\partial t^{\prime}}=\widehat{H}_{0}\left(\widehat{\mathbf{p}^{\prime}}, \mathbf{r}^{\prime}\right) \Psi\left(\mathbf{r}^{\prime}, t^{\prime}\right), \\
\widehat{H}_{0}\left(\widehat{\mathbf{p}^{\prime}}, \mathbf{r}^{\prime}\right)=m_{e} c^{2}+\frac{\widehat{\mathbf{p}}^{2}}{2 m_{e}}-\frac{e^{2}}{r^{\prime}},
\end{gathered}
$$

where proton is supposed to have a fixed position due to action of some nongravitational force on it. [Here $\widehat{\mathbf{p}^{\prime}}=$ $-i \hbar \partial / \partial \mathbf{r}^{\prime} ; m_{e}$ and $e$ are electron mass and charge, resp.] Let us discuss the approximation (1)-(3). First, in (1), (2), we take into account only terms of the order of $|\phi| / c^{2}$, which can be estimated as $10^{-9}$ near the Earth. Second, in (3), we disregard the so-called tidal effects. This means that we do not differentiate gravitational potential, $\phi$, with respect to electron coordinates, $\mathbf{r}$ and $\mathbf{r}^{\prime}$. In the next section, we estimate the tidal terms in the Hamiltonian, which, as will be shown, are of the order of $\left(r_{B} / R_{0}\right)\left|\phi / c^{2}\right|\left(e^{2} / r_{B}\right) \sim 10^{-17}\left|\phi / c^{2}\right|\left(e^{2} / r_{B}\right)$ in the Earth's gravitational field. [Here $r_{B}$ is a hydrogen atom typical "size" (i.e., Bohr's radius) and $R_{0}$ is the Earth's radius.] Third, we consider proton as a classical particle with mass $m_{p} \gg m_{e}$, whose position is fixed and kinetic energy is negligible. As usual, we treat the weak gravitation (1), (2), as a perturbation in the inertial coordinate system, corresponding to the coordinates $(x, y, z, t)$ in (2). By substitution of these coordinates in the Hamiltonian (3), it is easy to obtain the following effective electron Hamiltonian:

$$
\begin{aligned}
\widehat{H}(\widehat{\mathbf{p}}, \mathbf{r})= & m_{e} c^{2}+\frac{\widehat{\mathbf{p}}^{2}}{2 m_{e}}-\frac{e^{2}}{r}+m_{e} \phi \\
& +\left(3 \frac{\widehat{\mathbf{p}}^{2}}{2 m_{e}}-2 \frac{e^{2}}{r}\right) \frac{\phi}{c^{2}}
\end{aligned}
$$

and to rewrite it in more convenient form:

$$
\widehat{H}(\widehat{\mathbf{p}}, \mathbf{r})=m_{e} c^{2}+\frac{\widehat{\mathbf{p}}^{2}}{2 m_{e}}-\frac{e^{2}}{r}+\widehat{m}_{g}(\widehat{\mathbf{p}}, \mathbf{r}) \phi .
$$

We point out that, in (5), we introduce the following expression for electron passive gravitational mass operator:

$$
\begin{aligned}
\widehat{m}_{g}(\widehat{\mathbf{p}}, \mathbf{r})= & m_{e}+\left(\frac{\widehat{\mathbf{p}}^{2}}{2 m_{e}}-\frac{e^{2}}{r}\right) \frac{1}{c^{2}} \\
& +\left(2 \frac{\widehat{\mathbf{p}}^{2}}{2 m_{e}}-\frac{e^{2}}{r}\right) \frac{1}{c^{2}},
\end{aligned}
$$

which is equal to electron weight operator in the weak gravitational field (1). Note that, in (6), the first term is the bare electron mass, $m_{e}$, and the second term corresponds to the expected electron energy contribution to the mass operator, whereas the third term is the nontrivial virial contribution to the gravitational mass operator. We recall that the Hamiltonian (5) and (6) are derived for the case, where a hydrogen atom center of mass (i.e., proton) has a fixed 
position with respect to the Earth. In other words, it is supported in the gravitational field (1) by some nongravitational force. Now, suppose that the proton is dragged with small and constant (with respect to the Earth) velocity, $u \ll \alpha c$, by a spacecraft, where $\alpha$ is the fine structure constant and $\alpha c$ is a characteristic value of electron velocity in a hydrogen atom. In this case, we can use adiabatic approximation, which results in the following time-dependent perturbation for the electron Schrödinger equation [2, 5-8]:

$$
\widehat{V}(\widehat{\mathbf{p}}, \mathbf{r}, t)=\left(2 \frac{\widehat{\mathbf{p}}^{2}}{2 m_{e}}-\frac{e^{2}}{r}\right) \frac{\phi(R+u t)}{c^{2}}
$$

Note that we are interested in electron excitations, therefore, in the electron Hamiltonian (7), we keep only the virial term, which does not commute with the Hamiltonian, taken in the absence of gravitational field. Since the Hamiltonian (7) is time dependent it causes the appearance of electron excitations and, thus, the appearance of photon emission from a macroscopic ensemble of the atoms. It is important that the Hamiltonians (5)-(7) are not valid for the free falling atoms, where we have to introduce the so-called normal Fermi coordinates $[12,13]$. As a result free falling atoms "feel" only second derivatives of the gravitational potential $[12,13]$.

\section{The Most General Hamiltonian}

To strengthen our arguments, in this section, we derive our Hamiltonian (5) and (6) from the more general Hamiltonian of [14]. The latter Hamiltonian [14] is obtained from the Dirac equation in a curved space-time of General Relativity. In [14], completely different physical effect-the mixing effect between even and odd wave functions in a hydrogen atom (i.e., the so-called relativistic Stark effect)-is studied. It is important that it is studied not for the free falling atoms but for the atom, whose center of mass is supported by nongravitational force in the weak gravitational field (1). Note that the corresponding Hamiltonian is derived in $1 / c^{2}$ approximation, as in our case. The peculiarity of the calculations in [14] is that not only terms of the order of $\phi / c^{2}$ are calculated, as in our case, but also terms of the order of $\phi^{\prime} / c^{2}$, where $\phi^{\prime}$ is a symbolic derivative of $\phi$ with respect to relative electron coordinates in the atom. Note that, in accordance with the existing tradition, we call the latter terms tidal ones. Obtained in [14], the Hamiltonian (3.24) for the corresponding Schrödinger equation can be expressed as a sum of the following four terms:

$$
\widehat{H}(\widehat{\mathbf{P}}, \widehat{\mathbf{p}}, \widetilde{\mathbf{R}}, r)=\widehat{H}_{0}+\widehat{H}_{1}+\widehat{H}_{2}+\widehat{H}_{3},
$$

where

$$
\widehat{H}_{0}=m_{e} c^{2}+m_{p} c^{2}+\left[\frac{\widehat{\mathbf{P}}^{2}}{2\left(m_{e}+m_{p}\right)}+\frac{\widehat{\mathbf{p}}^{2}}{2 \mu}\right]-\frac{e^{2}}{r},
$$

$$
\begin{aligned}
& \widehat{H}_{1} \\
& =\left\{m_{e} c^{2}+m_{p} c^{2}+\left[3 \frac{\widehat{\mathbf{P}}^{2}}{2\left(m_{e}+m_{p}\right)}+3 \frac{\widehat{\mathbf{p}}^{2}}{2 \mu}-2 \frac{e^{2}}{r}\right]\right\} \\
& \quad \times\left(\frac{\phi-\mathbf{g} \widetilde{\mathbf{R}}}{c^{2}}\right), \\
& \widehat{H}_{2} \\
& =\frac{1}{c^{2}}\left(\frac{1}{m_{e}}-\frac{1}{m_{p}}\right)\left[-(\mathbf{g r}) \widehat{\mathbf{p}}^{2}+i \hbar \mathbf{g} \widehat{\mathbf{p}}\right] \\
& \quad+\frac{1}{c^{2}} \mathbf{g}\left(\frac{\widehat{\mathbf{s}_{\mathbf{e}}}}{m_{e}}-\frac{\widehat{\mathbf{s}}}{m_{p}}\right) \times \widehat{\mathbf{p}}+\frac{e^{2}\left(m_{p}-m_{e}\right)}{2\left(m_{e}+m_{p}\right) c^{2}} \frac{\mathbf{g r}}{r} \\
& \widehat{H}_{3} \\
& =\frac{3}{2} \frac{i \hbar \mathbf{g P}}{\left(m_{e}+m_{p}\right) c^{2}}+\frac{3}{2} \frac{\mathbf{g}\left(\mathbf{s}_{\mathbf{e}}+\mathbf{s} \mathbf{p}\right) \times \mathbf{P}}{\left(m_{e}+m_{p}\right) c^{2}} \\
& \quad-\frac{(\mathbf{g r})(\mathbf{P} \mathbf{p})+(\mathbf{P r})(\mathbf{g p})-i \hbar \mathbf{g P}}{\left(m_{e}+m_{p}\right) c^{2}}
\end{aligned}
$$

[Here, $\mathbf{g}=-G\left(M / R^{3}\right) \mathbf{R}$.] Let us describe notations in (8)-(12). Note that $\widetilde{\mathbf{R}}$ and $\mathbf{P}$ are position and momentum of a center of mass of the atom, correspondingly. On the other hand, $\mathbf{r}$ and $\mathbf{p}$ are relative electron position and momentum in the center of mass coordinate system; $\mu=m_{e} m_{p} /\left(m_{e}+m_{p}\right)$ is the reduced electron mass. As seen from (9), $\widehat{H}_{0}(\widehat{\mathbf{P}}, \widehat{\mathbf{p}}, r)$ corresponds to the Hamiltonian of a hydrogen atom in the absence of the external field. We point out that $\widehat{H}_{1}(\widehat{\mathbf{P}}, \widehat{\mathbf{p}}, \widetilde{\mathbf{R}}, r)$ corresponds to couplings of the bare electron and proton masses as well as their kinetic and potential energies with the gravitational field (1). The Hamiltonians $\widehat{H}_{2}(\widehat{\mathbf{p}}, \mathbf{r})$ and $\widehat{H}_{3}(\widehat{\mathbf{P}}, \widehat{\mathbf{p}}, \widetilde{\mathbf{R}}, r)$ describe the tidal effects.

Note that, in the previous section, we have semiquantitatively derived the Hamiltonian (5) and (6). Below, we strictly derive it from the more general Hamiltonian (8)-(12). First, we use the approximation, where $m_{p} / m_{e} \gg 1$, and, thus, we have $\mu=m_{e}$. This allows us to consider proton as a heavy classical particle. We can fix its position, $\widetilde{\mathbf{R}}=$ const, in coordinate system, corresponding to the source of the gravitational field (1), by putting $\mathbf{P}=0$ in the Hamiltonian (8)-(12). Therefore, we can disregard center of mass momentum and center of mass kinetic energy. Moreover, as seen from (12), $\widehat{H}_{3}(\widehat{\mathbf{P}}, \widehat{\mathbf{p}}, \mathbf{r})=0$ in this case. Now, let us estimate the first tidal term (11) in the Hamiltonian. We recall that $|\mathbf{g}| \simeq|\phi| / R_{0}$. It is important that $|\mathbf{r}| \sim \hbar /|\mathbf{p}| \sim r_{B}$ and $\mathbf{p}^{2} /\left(2 m_{e}\right) \sim e^{2} / r_{B}$ in a hydrogen atom. These allow us to evaluate the Hamiltonian (11) as $H_{2} \sim\left(r_{B} / R_{0}\right)\left(\phi / c^{2}\right)\left(e^{2} / r_{B}\right) \sim 10^{-17}\left(\phi / c^{2}\right)\left(e^{2} / r_{B}\right)$, which is $10^{-17}$ smaller than $H_{1} \sim\left(\phi / c^{2}\right)\left(e^{2} / r_{B}\right)$. Therefore, we can also disregard the first tidal term (11) in the total Hamiltonian (8)-(12). As a result, the Hamiltonian (8)-(12) can be rewritten in a familiar way:

$$
\widehat{H}(\widehat{\mathbf{p}}, r)=\widehat{H}_{0}(\widehat{\mathbf{p}}, r)+\widehat{H}_{1}(\widehat{\mathbf{p}}, r),
$$




$$
\begin{aligned}
& \widehat{H}_{0}(\widehat{\mathbf{p}}, r)=m_{e} c^{2}+\frac{\widehat{\mathbf{p}}^{2}}{2 m_{e}}-\frac{e^{2}}{r}, \\
& \widehat{H}_{1}(\widehat{\mathbf{p}}, r)=\left\{m_{e} c^{2}+\left[3 \frac{\widehat{\mathbf{p}}^{2}}{2 m_{e}}-2 \frac{e^{2}}{r}\right]\right\}\left(\frac{\phi}{c^{2}}\right),
\end{aligned}
$$

where we place the proton at the point $\widetilde{R}=R$. Thus, we can make a conclusion that the Hamiltonian (13)-(15), derived in this section, exactly coincides with the Hamiltonian, semiquantitatively derived by us earlier [2, 5-8] [see (5), (6)]. Now, suppose that spacecraft moves proton from a hydrogen atom with constant and small velocity, $u \ll \alpha c$; then the second tidal term (12) in the total electron Hamiltonian is not zero. Nevertheless, it is easy to show that it can be estimated as $H_{3} \sim\left(r_{B} / R_{0}\right)\left(\phi / c^{2}\right)\left(e^{2} / r_{B}\right) \sim 10^{-17}\left(\phi / c^{2}\right)\left(e^{2} / r_{B}\right)$ and, thus, can be omitted. As a result, in adiabatic approximation, we obtain from (15) the time-dependent perturbation (7).

\section{What Is Right and What Is Wrong?}

As earlier as in [5], we concluded that the effect suggested by us was very general. In particular, we proposed [5] to use it not only in atomic physics, but also in condensed matter physics $[5,10]$, nuclear physics $[1,5]$, and elementary particle physics $[1,5]$. Here, we recall the physical meaning of the effect. Some quantum macroscopic system is placed in spacecraft and dragged with small constant velocity in an external gravitational field. In this case, due to nonhomogeneous squeezing of space by the field, there appear some quantum excitations in the system, which result in emission of photons $[2,5-8]$, phonons [10], pions [1], or some other particles. The experimental task is to detect these particles. We pay attention that, in all our previous works $[2,5-8,10]$ as well as in the previous sections of the current paper, we consider the case, where center of mass of a composite quantum system is dragged by spacecraft. It is important that it is dragged by means of nongravitational force with constant velocity with respect to source of gravity. We claim that the extension of our effect to free falling bodies, performed in the comment [1], is not legitimate. It is clearly seen from papers $[12,13]$, where examples of a free falling hydrogen atom is considered and the Fermi normal coordinates are used. As stressed in $[12,13]$, the free falling atoms "feel" only second derivative of the metric (1) and, thus, cannot exhibit our effect. This is also true for nuclear versions of a free falling experiment, considered in the comment [1]. To summarize our effect does not have to be observed in "free fall" experiments, discussed in [1]. Therefore, the central statement of comment [1], that the nucleus experiments contradict our effect $[2,5-8,10]$, is incorrect.

\section{Conflicts of Interest}

The author declares that there are no conflicts of interest regarding the publication of this paper.

\section{Acknowledgments}

The authors are thankful to N. N. Bagmet (Lebed), V. A. Belinski, Steven Carlip, Douglas Singleton, Elias Vagenas, and V. E. Zakharov for useful discussions.

\section{References}

[1] B. Crowell, "Comment on 'Does the Equivalence between Gravitational Mass and Energy Survive for a Composite Quantum Body?"' Advances in High Energy Physics, vol. 2016, Article ID 2958579, 4 pages, 2016.

[2] A. G. Lebed, "Does the equivalence between gravitational mass and energy survive for a composite quantum body?" Advances in High Energy Physics, vol. 2014, Article ID 678087, 10 pages, 2014.

[3] R. Colella, A. W. Overhauser, and S. A. Werner, "Observation of gravitationally induced quantum interference," Physical Review Letters, vol. 34, no. 23, pp. 1472-1474, 1975.

[4] V. V. Nesvizhevsky, H. G. Börner, A. K. Petukhov et al., "Quantum states of neutrons in the Earth's gravitational field," Nature, vol. 415, no. 6869, pp. 297-299, 2002.

[5] A. G. Lebed, "Is gravitational mass of a composite quantum body equivalent to its energy?" Central European Journal of Physics, vol. 11, no. 8, pp. 969-976, 2013.

[6] A. G. Lebed, "Breakdown of the equivalence between gravitational mass and energy for a composite quantum body," Journal of Physics: Conference Series, vol. 490, no. 1, Article ID 012154, 2014.

[7] A. G. Lebed, "Breakdown of the equivalence between passive gravitational mass and energy for a quantum body," in Proceedings of the MG13 Meeting on General Relativity, R. Ruffini, R. Jantzen, and K. Rosquist, Eds., pp. 1953-1955, World Scientific, Stockholm, Sweden, 2015.

[8] A. G. Lebed, "Breakdown of the equivalence between gravitational mass and energy for a quantum body: theory and suggested experiments," International Journal of Modern Physics. D. Gravitation, Astrophysics, Cosmology, vol. 24, no. 11, Article ID 1530027, 23 pages, 2015.

[9] A. G. Lebed, "Breakdown of the equivalence between active gravitational mass and energy for a quantum body," Journal of Physics: Conference Series, vol. 738, no. 1, Article ID 012036, 2016.

[10] A. G. Lebed, "Inequivalence between gravitational mass and energy due to quantum effects at microscopic and macroscopic levels," International Journal of Modern Physics D. Gravitation, Astrophysics, and Cosmology, 2017.

[11] W. K. Misner, S. Thorne, and J. A . Wheeler, Gravitation, W. H. Freeman and Co, New York, NY, USA, 1973.

[12] L. Parker, "One-electron atom in curved space-time," Physical Review Letters, vol. 44, no. 23, pp. 1559-1562, 1980.

[13] F. Pinto, "Rydberg atoms in curved space-time," Physical Review Letters, vol. 70, no. 25, pp. 3839-3843, 1993.

[14] E. Fischbach, B. S. Freeman, and W.-K. Cheng, "Generalrelativistic effects in hydrogenic systems," Physical Review D, vol. 23, no. 10, pp. 2157-2180, 1981. 

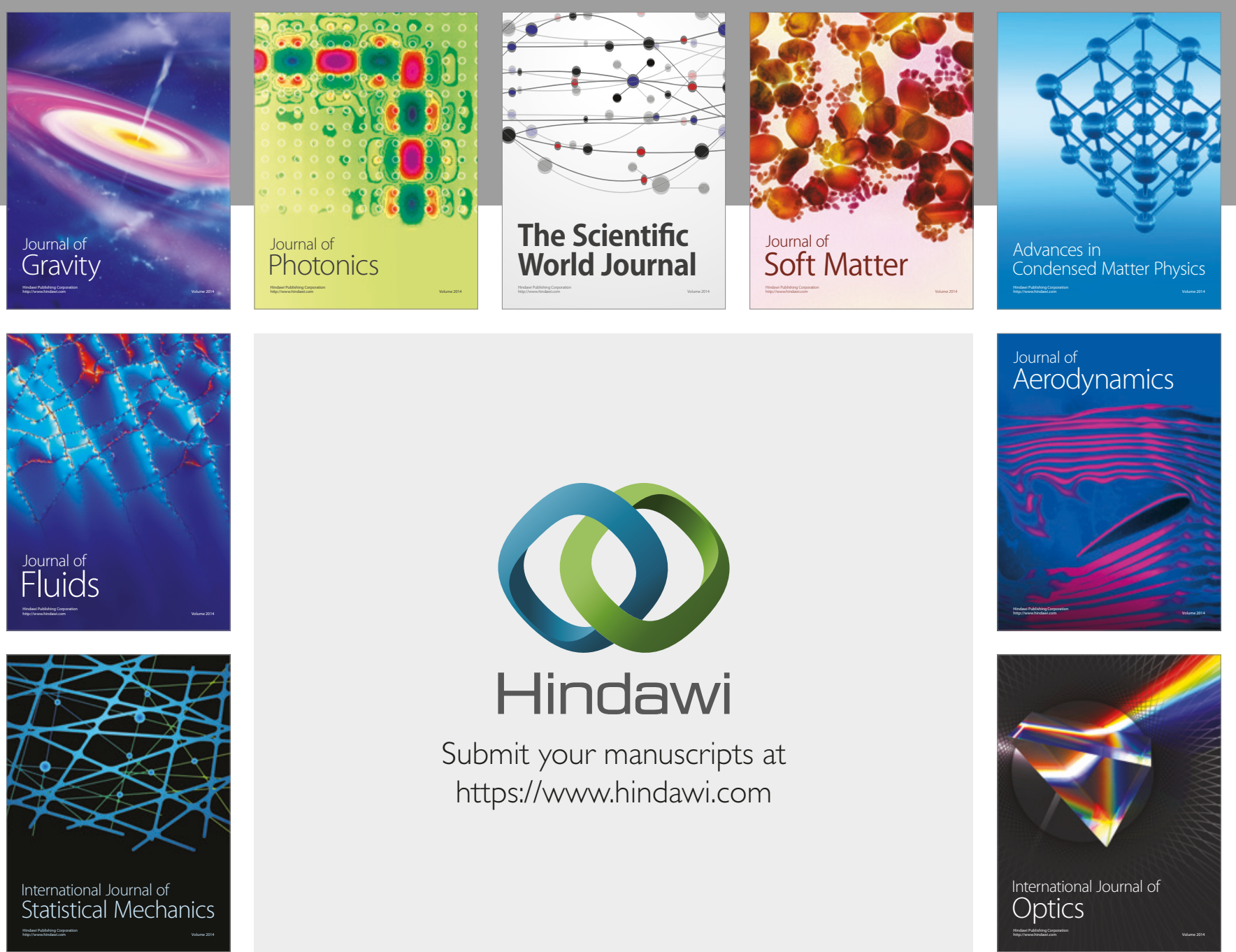

Submit your manuscripts at

https://www.hindawi.com
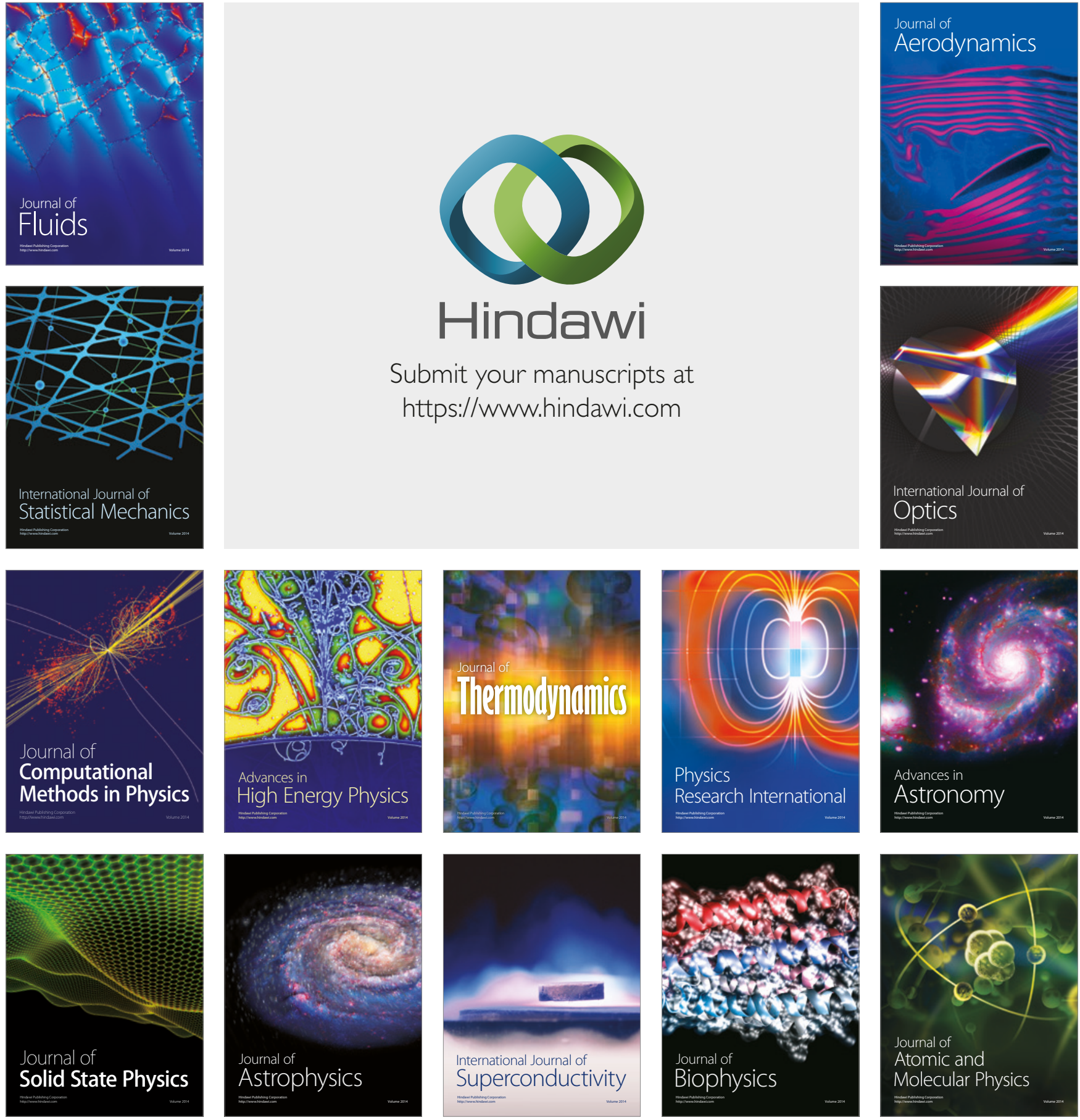